\title{
EFECTO DE UN SUPLEMENTO NUTRICIONAL A BASE DE LÍPIDOS EN LOS NIVELES DE HEMOGLOBINA E INDICADORES ANTROPOMÉTRICOS EN NIÑOS DE CINCO DISTRITOS DE HUÁNUCO, PERÚ
}

\author{
Alejandro Vargas-Vásquez ${ }^{1, a}$, Ricardo Bado $^{2, b}$, Lorena Alcázar ${ }^{3, c}$, Oscar Aquino ${ }^{4, d}$, Amelia Rodríguez $^{5, b, e}$ \\ José Pedro Novalbos ${ }^{5, \mathrm{~b}, \mathrm{f}}$
}

\begin{abstract}
RESUMEN
Objetivos. Determinar el efecto del consumo de un suplemento nutricional a base de lípidos (LNS) sobre los niveles de hemoglobina, anemia e indicadores antropométricos, en niños de seis a once meses de edad de cinco distritos en la provincia de Ambo del departamento de Huánuco, Perú. Materiales y métodos. Se efectuó un estudio preexperimental, prepost. La población de estudio fueron niños de seis a once meses de edad de 19 establecimientos de salud de cinco distritos con muy alta vulnerabilidad a la desnutrición crónica de la provincia de Ambo, Huánuco; se obtuvieron datos de 147 niños que recibieron suplemento nutricional a base de lípidos (LNS) desde los seis a los once meses de edad. Resultados. El promedio de hemoglobina se incrementó significativamente en $0,67 \mathrm{~g} / \mathrm{dL}(p<0,05)$, la proporción de anemia se redujo en 27 puntos porcentuales $(p<0,001)$ y el promedio del puntaje $z$ para talla/edad disminuyó en $0,12(p<0,05)$ y no se encontraron diferencias significativas para los puntajes $z$ de peso/edad y peso/talla al finalizar el estudio. EI LNS tuvo una adherencia, superior al $90 \%$, y menos del $10 \%$ de los niños del estudio reportaron molestias durante el consumo. Conclusiones. La suplementación con LNS logro mejorar los niveles de hemoglobina y reducir la prevalencia de anemia en niños menores de doce meses, por lo que podría constituir una alternativa efectiva para prevenir y controlar la anemia infantil.
\end{abstract}

Palabras clave: Alimentación suplementaria; Anemia; Hemoglobinas (fuente: DeCS BIREME).

\section{EFFECTS OF A LIPID-BASED NUTRIENT SUPPLEMENT ON HEMOGLOBIN LEVELS AND ANTHROPOMETRIC INDICATORS IN CHILDREN FROM FIVE DISTRICTS IN HUÁNUCO PERU}

\begin{abstract}
Objectives. To determine the effect of consumption of a lipid-based nutrient supplement (LNS) on hemoglobin levels, anemia and anthropometric indicators in children aged six to eleven months old in five districts in the province of Ambo in the region of Huanuco, Peru. Materials and methods. A pre-experimental, pre-post study was performed. The study population included children aged six to eleven months old in 19 health facilities in five districts with very high vulnerability to chronic malnutrition in the province of Ambo, Huanuco. Data from 147 children who received the lipid-based nutrient supplement (LNS) from six to eleven months old were obtained. Results. The mean hemoglobin significantly increased by $0.67 \mathrm{~g} / \mathrm{dL}(\mathrm{p}<0.05)$, the proportion of anemia dropped by 27 percentage points $(p<0.001)$ and the mean $z$ score for height/ age decreased by $0.12(p<0.05)$. No significant differences for $z$ scores of weight/age and weight/height at the end of the study were found. The LNS had an adherence greater than $90 \%$, and less than $10 \%$ of children in the study reported discomfort during consumption. Conclusions. LNS supplementation achieved improved hemoglobin levels and reduced the prevalence of anemia in children under twelve months, which might constitute an effective alternative to prevent and control childhood anemia.
\end{abstract}

Key words: Supplementary feeding; Anemia; Hemoglobin (source: MeSH NLM).

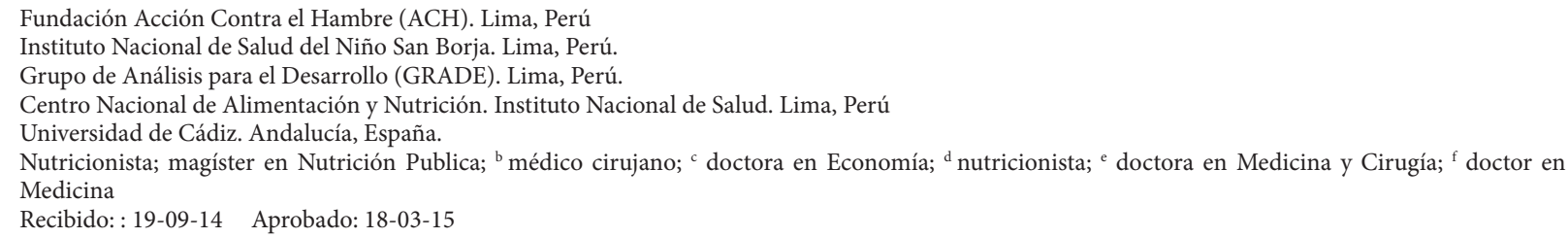

Nutricionista; magíster en Nutrición Publica; ${ }^{\mathrm{b}}$ médico cirujano; ${ }^{\mathrm{c}}$ doctora en Economía; ${ }^{\mathrm{d}}$ nutricionista; ${ }^{\mathrm{e}}$ doctora en Medicina y Cirugía; ${ }^{\mathrm{f}}$ doctor en Medicina

Recibido: : 19-09-14 Aprobado: 18-03-15

Citar como: Vargas-Vásquez A, Bado R, Alcázar L, Aquino O, Rodríguez A, Novalbos JP. Efecto de un suplemento nutricional a base de lípidos en los niveles de hemoglobina e indicadores antropométricos en niños de cinco distritos de Huánuco, Perú. Rev Peru Med Exp Salud Publica. 2015;32(2): 237-44. 


\section{INTRODUCCIÓN}

La desnutrición y las carencias de micronutrientes causan retraso en el crecimiento y desarrollo durante la infancia; y menor productividad en los individuos cuando llegan a la edad adulta ${ }^{(1,2)}$. Durante la niñez, la desnutrición crónica afecta las tasas de morbilidad y mortalidad infantil y, a largo plazo, impacta negativamente en el crecimiento lineal, el desempeño escolar, el estado de salud y la productividad laboral (3). Los costos de la desnutrición afectan al individuo y a la sociedad de tal forma que genera un círculo vicioso lo cual constituye causa y consecuencia de la pobreza ${ }^{(4)}$.

En el Perú, la desnutrición crónica en niños menores de cinco años, tiene una tendencia sostenida de reducción desde el año 2007 hasta el presente año, variando de 28,4 a $14,1 \%$; sin embargo, esta disminución no es homogénea en todos los ámbitos, de tal forma que la brecha entre el área urbana y rural es de 21 puntos porcentuales ${ }^{(5,6)}$. Algunos estudios muestran que la reducción de la desnutrición crónica ha ocurrido con mayor énfasis entre los niños "más privilegiados" es decir en el área urbana, con madres más educadas y de quintiles de pobreza superiores ${ }^{(7,8)}$.

Por su parte, la proporción de anemia en niños de 6 a 36 meses de edad ha tenido un comportamiento variable, si bien se produjo una reducción de 15 puntos porcentuales entre los años 2007 y $2011^{(5,9)}$ para el periodo 2011-2014 se observa un aumento de 8 puntos porcentuales ${ }^{(6,9)}$ a pesar de que en los últimos tres años se ha implementado a nivel nacional la suplementación con multimicronutrientes en polvo (MNP). Algunos autores han reportado barreras en la suplementación con MNP, dado que había dificultades en la distribución, uso y consumo del suplemento ${ }^{(10)}$.

Recientes estudios han documentado la efectividad de suplementos nutricionales a base de lípidos (LNS por Lipid-based Nutrient Suplement), sobre el crecimiento lineal ${ }^{(11)}$ y reducción de la anemia $(12,25)$ en comparación con otros suplementos nutricionales. EI LNS es un suplemento alimenticio complementario listo para consumir, que ha sido desarrollado para las necesidades de niños de 6 a 24 meses de edad. Su presentación es un sachet de $20 \mathrm{~g}$ y sus ingredientes son maní, aceite y leche, por lo que su composición presenta ácidos grasos esenciales y niveles adecuados de vitaminas y minerales ${ }^{(13)}$. Este suplemento también ha mostrado resultados positivos en adherencia al consumo, aceptabilidad y poca frecuencia de efectos adversos ${ }^{(14,15)}$. Desde esa perspectiva, el objetivo del presente estudio es determinar el efecto del consumo de un suplemento nutricional a base de lípidos (LNS) sobre los niveles de hemoglobina, anemia e indicadores antropométricos de niños de 6 a 11 meses de edad de cinco distritos en la provincia de Ambo en el departamento de Huánuco.

\section{MATERIALES Y MÉTODOS}

\section{DISEÑO DEL ESTUDIO}

Se efectuó un estudio preexperimental pre-post (16), durante los meses de julio de 2013 a marzo de 2014 en la provincia de Ambo, región Huánuco, Perú. La población de estudio estuvo compuesta por niños de 6 a 11 meses de edad que recibían sus atenciones de salud en alguno de los 19 establecimientos de salud del Ministerio de Salud (MINSA) que se ubican en los distritos de Cayna, Colpas, Huacar, San Francisco y San Rafael ubicados en la provincia de Ambo, Huánuco. Estos distritos se eligieron por su calificación de muy alta vulnerabilidad a la desnutrición crónica ${ }^{(17)}$ y por tener condiciones similares en pobreza, población rural, características de la vivienda, acceso a servicios de agua, desagüe, electricidad, analfabetismo, inasistencia a centros educativos y altitud.

El cálculo del tamaño de muestra se realizó considerando un incremento de hemoglobina de $0,46 \mathrm{~g} / \mathrm{dL}{ }^{(11)}$ en el mismo grupo luego de 6 meses de suplementación, una significancia de $95 \%$ y un poder estadístico de 0,90 , con estos valores se estimó necesario un tamaño muestral mínimo de 63 niños. Durante un período de nueve meses se enrolaron a todos los niños que cumplieron seis meses de edad y que recibieron sus controles de crecimiento y desarrollo (CRED) en alguno de los 19 establecimientos de salud seleccionados. No se incluyeron niños con enfermedades crónicas, congénitas o severas que impidieran un crecimiento o metabolismo normal, ni tampoco niños que hubieran sido producto de embarazos gemelares o de mellizos; ni niños con menos de $2500 \mathrm{~g}$ de peso al nacer. De julio de 2013 a marzo de 2014, se enrolaron 160 niños, considerando las posibles pérdidas en el seguimiento (constituyendo un censo de los niños de seis meses en el ámbito de estudio). Al finalizar el estudio se contaba con datos de 147 niños, por lo que se realizó un análisis post hoc determinándose que para este tamaño muestral, el poder estadístico era de 0,99 y un error alfa de 0,05.

\section{INTERVENCIÓN}

Los niños enrolados en el estudio recibieron mensualmente, por un período de 6 meses, 30 sachets de un suplemento nutricional a base de lípidos (LNS), 
denominado Nutributter®. Diariamente, los niños debían consumir 01 sachet de LNS, hasta completar 180 en 6 meses. El consumo del LNS podía ser directo del sachet o mezclado con alguna comida. El contenido de un sachet de $20 \mathrm{~g}$, Nutributter contiene: $108 \mathrm{kcal}$; 2,6 $\mathrm{g}$ de proteínas; $7 \mathrm{~g}$ de grasa. Vitaminas: vitamina A (0,4 mg), vitamina B1 (0,3 mg), vitamina B6 (0,3 $\mathrm{mg})$, niacina (1,8 $\mathrm{mg})$, vitamina $\mathrm{C}(30 \mathrm{mg})$, vitamina B2 (0,4 mg), vitamina B12 (0,5 mg), ácido fólico (80 mg). Minerales: calcio (100 mg), potasio (152 mg), zinc (4 $\mathrm{mg}$ ), hierro (9 $\mathrm{mg})$, selenio (10 $\mathrm{mg})$, fósforo (82 mg), magnesio (16 mg), cobre $(0,2 \mathrm{mg})$, yodo $(90 \mathrm{mg}) \mathrm{y}$ manganeso $(0,08 \mathrm{mg})$.

\section{RECOLECCIÓN DE INFORMACIÓN}

A los seis meses de edad cumplidos (antes de iniciar la recepción del suplemento alimentario) y a los doce meses de edad cumplidos, se determinó el peso, la talla y el nivel de hemoglobina de los niños. Para la toma de medidas antropométricas del peso y talla se usaron balanzas digitales con precisión de $100 \mathrm{~g}$ y tallímetros portátiles modelo OMS con precisión de $0,1 \mathrm{~cm}$. Para la evaluación de los niveles de hemoglobina de los niños, se utilizó el método de colorimetría en sangre capilar con el fotómetro portátil HemoCue ${ }^{\circledR}{ }^{(18)}$. Los monitores de campo del estudio se encargaron de efectuar las mediciones antropométricas y bioquímicas, para lo cual fueron previamente estandarizados y capacitados según las técnicas recomendadas por el Instituto Nacional de Salud (INS) ${ }^{(18,19)}$.

Con los datos de peso, talla y edad se calcularon los siguientes indicadores antropométricos: talla para la edad, peso para la edad y peso para la talla; y con ello se determinó desnutrición crónica, global y aguda respectivamente, cuando presentaron menos de -2 desviaciones estándar del puntaje $Z$ según patrón de referencia OMS $2006{ }^{(20)}$. El cálculo de los indicadores antropométricos se realizó en el programa Anthro de OMS. Se definió anemia cuando se encontró valores menores de $11 \mathrm{~g} / \mathrm{dL}$ de hemoglobina ajustada por altitud, considerando la clasificación recomendada por la OMS ${ }^{(18,21)}$.

La altitud usada para la corrección del nivel de hemoglobina se determinó en la vivienda de cada niño, para ello se utilizaron altímetros marca SUUNTO. Además, se recolectó mensualmente información sobre el consumo del LNS, aceptabilidad, dilución intrafamiliar y molestias reportadas por las madres. Se consideraron como variables respuesta la modificación inicial-final de los puntajes $Z$ de los indicadores antropométricos (peso/ talla, talla/edad, peso/edad); la concentración sérica de hemoglobina, así como la adherencia y aceptabilidad del LNS. La adherencia se definió como la razón de sachets de LNS consumidos entre sachets de LNS recibidos, multiplicado por 100. La frecuencia de consumo del suplemento fue determinado por el número de días que usualmente el niño consumió el suplemento durante la última semana y se consideró que el suplemento tenía aceptabilidad cuando la cantidad que el niño consumía diariamente era más de la mitad o todo el sachet en el mes. Asimismo, se definió como dilución intrafamiliar al consumo del LNS por otro miembro de la familia que no era el niño en estudio.

La entrega del LNS se realizó mensualmente en los consultorios del Área Niño de los establecimientos de salud del MINSA, cuando los niños asistían al control de crecimiento y desarrollo (CRED). El personal de salud responsable de distribuir el suplemento y brindar atención en salud fue capacitado para dar consejería sobre el adecuado uso y consumo del LNS.

Los monitores de campo realizaron visitas domiciliarias de seguimiento mensual desde los 7 a los 12 meses de edad del niño y preguntaban a las madres 0 cuidadores por la frecuencia de consumo, cantidad consumida del sachet, la dilución intrafamiliar, la forma en que fue consumido y qué molestias se presentaron. Al finalizar el sexto mes de seguimiento de cada niño, el monitor asignado recabó la información del número de sachets entregados en el establecimiento de salud; además, en la última visita domiciliaria solicitó a la madre los sachets llenos de LNS que habían sido consumidos, para contabilizar el consumo total, previo descuento del número de sachets consumidos por otros familiares que no eran los niños en estudio. Antes de dar el alta al seguimiento de los niños que completaron el estudio, se determinó el peso, la talla y el nivel de hemoglobina.

\section{ANÁLISIS ESTADÍSTICO}

La base de datos fue generada por digitación en el software CSPro v.5.0 y luego de verificar la consistencia de los datos se exportó al programa estadístico SPSS V.17. Se calcularon proporciones para las variables categóricas mientras que para las variables numéricas se calcularon las medias y la desviación estándar con previa verificación de normalidad usando la prueba de Shapiro Wilk. También se calculó la prevalencia y los intervalos de confianza. Además, se compararon y calcularon las diferencias porcentuales y de promedios en los resultados iniciales y finales de los puntajes $Z$ de los indicadores antropométricos, hemoglobina y anemia, para lo cual se empleó la prueba $t$ de Student para muestras pareadas, y test de Mc Nemar para datos categóricos. 


\section{CONSIDERACIONES ÉTICAS}

Los padres de los niños menores de seis meses fueron visitados en sus hogares con el fin de explicarles el protocolo de estudio e invitarlos a que sus niños participen del estudio. Se precisó que la participación del niño era voluntaria y que podían retirarse del estudio si lo requerían. Los padres que aceptaron ingresar al estudio firmaron la hoja de consentimiento informado. El protocolo de investigación y el consentimiento informado fueron aprobados por el Comité de Ética de la Asociación Benéfica PRISMA y el estudio fue autorizado por el INS.

\section{RESULTADOS}

De julio de 2012 a marzo de 2013 se enrolaron, en los 19 establecimientos de salud seleccionados, 160 niños y niñas que cumplieron los criterios de inclusión, se perdieron 13 individuos en el proceso $(8,1 \%)$ y terminaron el seguimiento 147 . Todos iniciaron el estudio con 6 meses de edad; según sexo el 44,2\% (65) eran varones, casi la totalidad $(99,3 \%)$ de los individuos recibió lactancia materna; respecto a programas sociales o intervenciones, un $84 \%$ era beneficiario de alguna intervención y solo el $26,5 \%$ pertenecía al programa JUNTOS. EI $55 \%$ de las madres realizó estudios en el nivel primario, el $24,5 \%$ en el nivel secundario, el $18,4 \%$ no tenía estudios y un $2 \%$ realizó estudios superiores. El promedio de personas en el hogar fue de 5,15 $\pm 1,73$; $y$ el domicilio se encontraba, en promedio, a 3089,4 metros $\pm 83,52$.
Al finalizar los seis meses de suplementación, el 78,2\% de los niños (115) consumieron 180 sachets de LNS, es decir, tuvo una adherencia de $100 \%$; en el resto de niños la adherencia fue superior a $90 \%$. Respecto a la frecuencia de consumo del suplemento, la Tabla 1 muestra que más del $80 \%$ de los niños consumían siete sachets a la semana, observándose la proporción más baja al tercer mes $(86,4 \%)$ y la mayor al sexto mes $(97,4 \%)$; en cuanto a la aceptabilidad, considerada como el consumo diario de más de la mitad o todo el sachet de LNS en el mes, se observa un aumento de $66 \%$ al inicio, hasta $89 \%$ al sexto mes de suplementación. Por su parte, la dilución intrafamiliar muestra un descenso de $5,4 \%$ al inicio a $2 \%$ al final de la suplementación. Este mismo comportamiento se aprecia en la proporción de madres que reportaron la aparición de molestias en los niños suplementados que varió de 8,2 a 1,4\% en el mismo período.

Los indicadores antropométricos a los 6 y 12 meses se muestran en la Tabla 2 . El índice $Z$ de talla/edad se redujo de -0,99 (IC 95\%: -1,14, -0,82) a -1,12 (IC 95\%: -1,26, $-0,95)$ mientras que el número de niños con desnutrición crónica pasó de 20 a 24 en el periodo de seguimiento. En cuanto al índice $Z$ de peso/edad no se encontró diferencias significativas en los dos evaluaciones, aunque 3 niños mejoraron su condición de desnutridos globales a normales al finalizar la suplementación. Si bien se aprecia una disminución significativa de la media del puntaje $\mathrm{Z}$ peso/talla, los datos también muestran que al inicio había 2 niños con desnutrición aguda y al finalizar los seis meses solo había 1 niño en esa condición. Respecto al nivel de hemoglobina se encontró que la

Tabla 1. Características de consumo y aceptabilidad del un suplemento nutricional a base de lípidos en niños de seis a once meses de la provincia de Ambo, Huánuco

\begin{tabular}{|c|c|c|c|c|c|c|}
\hline & Mes 1 & Mes 2 & Mes 3 & Mes 4 & Mes 5 & Mes 6 \\
\hline \multicolumn{7}{|c|}{ Sachets consumidos en la semana (\%) } \\
\hline 7 sachets & $131(89,1)$ & $128(87,1)$ & $127(86,4)$ & $131(89,1)$ & $129(87,8)$ & $143(97,2)$ \\
\hline 4-6 sachets & $11(7,5)$ & $14(9,5)$ & $15(10,2)$ & $10(6,8)$ & $13(8,7)$ & $1(0,7)$ \\
\hline $1-3$ sachets & $5(3,4)$ & $5(3,4)$ & $5(3,4)$ & $6(4,1)$ & $4(2,8)$ & $3(2,1)$ \\
\hline Ningún sachet & $0(0,0)$ & $0(0,0)$ & $0(0,0)$ & $0(0,0)$ & $1(0,7)$ & $0(0,0)$ \\
\hline \multicolumn{7}{|c|}{ Cantidad del sachet consumido (\%) } \\
\hline Todo & $76(51,7)$ & $89(60,5)$ & $99(67,3)$ & $103(70,1)$ & $103(70,5)$ & $127(86,4)$ \\
\hline Más de la mitad & $21(14,3)$ & $17(11,6)$ & $6(4,1)$ & $11(7,5)$ & $13(8,9)$ & $5(3,4)$ \\
\hline La mitad o menos & $50(34,0)$ & $41(27,9)$ & $42(28,5)$ & $33(22,4)$ & $30(20,6)$ & $15(10,2)$ \\
\hline \multicolumn{7}{|c|}{ Dilución intrafamiliar (\%) } \\
\hline No & $139(94,6)$ & $141(95,9)$ & $143(97,3)$ & $141(95,9)$ & $138(93,9)$ & $144(98,0)$ \\
\hline Sí & $8(5,4)$ & $6(4,1)$ & $4(2,7)$ & $6(4,1)$ & $9(6,1)$ & $3(2,0)$ \\
\hline \multicolumn{7}{|c|}{ Molestias reportadas por la madre (\%) } \\
\hline Ninguna & $135(91,8)$ & $133(90,5)$ & $142(96,6)$ & $143(97,3)$ & $143(97,3)$ & $145(98,6)$ \\
\hline $\begin{array}{l}\text { Le hizo daño (dia- } \\
\text { rrea, vómitos) }\end{array}$ & $4(2,7)$ & $4(2,7)$ & $1(0,7)$ & $3(2,0)$ & $0(0,0)$ & $2(1,4)$ \\
\hline No le gusta el sabor & $7(4,8)$ & $10(6,8)$ & $4(2,7)$ & $1(0,7)$ & $4(2,7)$ & $0(0,0)$ \\
\hline Otros & $1(0,7)$ & $0(0,0)$ & $0(0,0)$ & $0(0,0)$ & $0(0,0)$ & $0(0,0)$ \\
\hline
\end{tabular}


Tabla 2. Cambio de índices antropométricos e indicadores bioquímicos después de 6 meses de consumo de un suplemento nutricional a base de lípidos

\begin{tabular}{|c|c|c|c|c|}
\hline & Medición inicial & Medición final & Diferencia & Valor $p$ \\
\hline $\mathrm{Z}$ talla/edad (IC 95\%) & $-0,99(-1,14,-0.82)$ & $-1,12(-1,26,-0,95)$ & $0,12(-0,26,-0,01)$ & $0,037^{*}$ \\
\hline Niños con z talla/edad $<-2 \mathrm{Z}(\%)$ & $20(13,7)$ & $24(16,4)$ & $4(2,7)$ & ** \\
\hline Z pesoledad (IC 95\%) & $-0,12(-0,29,0,03)$ & $-0,17(-0,33,-0,05)$ & $0,05(-0,17,0,04)$ & 0,24 \\
\hline Niños con z peso/edad < -2 Z (\%) & $6(4,1)$ & $3(2,0)$ & $3(2,1)$ & ** \\
\hline Z peso/talla (IC 95\%) & $0,71(0,54,0,88)$ & $0,49(0,36,0,63)$ & $0,22(-0,36,-0,08)$ & $0,003^{*}$ \\
\hline Niños con z peso/talla $<-1 \mathrm{Z}(\%)$ & $4(2,7)$ & $5(3,4)$ & $1(0,7)$ & ** \\
\hline Niños con z peso/talla $<-2 \mathrm{Z}(\%)$ & $2(1,4)$ & $1(0,7)$ & $1(0,7)$ & ** \\
\hline Hemoglobina (g/dL) (IC 95\%) & $10,26(10,11,10,42)$ & $10,93(10,79,11,07)$ & $0,67(0,48,0,84)$ & $<0,001^{*}$ \\
\hline Niños sin anemia (\%) & $30(20,4)$ & $70(47,6)$ & $40(27,2)$ & $<0,001^{*}$ \\
\hline Anemia leve (\%) & $61(41,5)$ & $58(39,5)$ & $3(2,0)$ & ** \\
\hline Anemia moderada (\%) & $56(38,1)$ & $19(12,9)$ & $37(25,2)$ & $<0,001^{*}$ \\
\hline
\end{tabular}

${ }^{*} \mathrm{p}<0,05 ;{ }^{* *}$ Valor no determinado, por el reducido número de casos

media aumento significativamente de 10,26 (IC 95\%: $10,11,10,42)$ a 10,93 (IC 95\%: 10,79, 11,07) y que la prevalencia de anemia tuvo una reducción significativa de 27 puntos porcentuales en el periodo de estudio.

La Figura 1 muestra el cambio de la prevalencia de anemia en los niños que terminaron el seguimiento hasta los 12 meses, se observa que del total de niños que al inicio tenían anemia moderada $(n=56)$, el $37,5 \%$ terminó en condición normal, el 35,7\% pasó de anemia moderada a leve y un $26,8 \%$ permaneció en anemia moderada; del total de niños con anemia leve a los 6 meses ( $n=61$ ) un 45,9\% terminó el seguimiento sin anemia, el 50,8\% permaneció en la misma condición y un 3,3\% desarrolló anemia moderada. Por la parte del total de niños que inició el estudio sin anemia $(n=30)$ el $70 \%$ permaneció en esta condición, un 23,3\% terminó con anemia leve y el 6,7\% en anemia moderada.

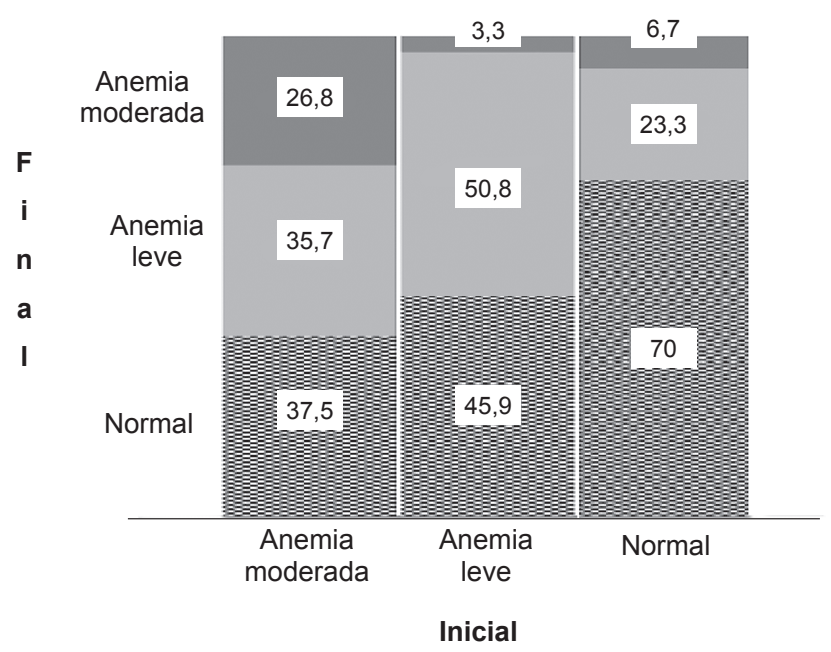

Figura 1. Variación de prevalencia según tipo de anemia y condición normal después del consumo de un suplemento nutricional a base de lípidos

\section{DISCUSIÓN}

El presente estudio muestra el efecto del consumo del LNS en una población de niños de seis hasta los once meses de edad de la provincia de Ambo, Huánuco. Se encontró efecto para mejorar los niveles de hemoglobina y reducir la prevalencia de anemia; así como una alta adherencia y aceptabilidad, poca dilución intrafamiliar y bajos reportes de molestias durante el consumo.

Este es el primer estudio en el Perú para conocer el efecto del LNS sobre indicadores antropométricos y hemoglobina. Otros estudios encontraron impacto del LNS en el crecimiento lineal y los niveles de hemoglobina. Así, lannotti et al. encontraron que un LNS mejoró el crecimiento infantil con resultados que se mantuvieron 6 meses después de la intervención (22); por su parte Phuka et al. no encontraron diferencias significativas en aumento de peso y talla en niños al comparar el LNS con harina de maíz; sin embargo, reportaron menor incidencia de desnutrición crónica severa al impulsar el crecimiento lineal de los niños tempranamente desfavorecidos (23). Este mismo grupo de investigadores encontraron, posteriormente, que el LNS frente a un grupo control tuvo un impacto positivo sobre la mejora de los niños con insuficiencia ponderal moderada ( $Z$ score $P / T: 0,33){ }^{24}$. Por otro lado, Adu-Afarwuah et al., realizaron un ensayo aleatorizado en Ghana, en niños de seis a once meses, comparando la eficacia y aceptabilidad de Sprinkles, tabletas Nutritab y el LNS-Nutributter, encontrando que los niveles de hemoglobina en los grupos que recibieron Nutri Tab o Nutributter fueron significativamente mayores con respecto al grupo intervenido, lo cual no sucedió con el grupo de Sprinkles ${ }^{(25)}$. También se encontró que los tres grupos suplementados tuvieron resultados positivos en el desarrollo motor de los niños, pero solo el Nutributter tuvo efectos positivos en el crecimiento lineal, con promedio de índices de talla para la edad (T/E) de $-0,20 \pm 0,54$, en 
comparación al promedio del Nutritab de $-0,39 \pm 0,54$ o del grupo combinado Nutritab+Sprinkles $(-0,38 \pm 0,54)$ y el grupo no intervenido $(-0,40 \pm 1,0)^{(11)}$.

Acerca del efecto del LNS sobre el crecimiento lineal, en este estudio se encontró una reducción de 0,12 del puntaje $Z$ de talla/edad (agravamiento de la situación), esta caída es menor a lo esperado en poblaciones con estas características, así, usando la base de datos del Sistema de Información del Estado Nutricional del MINSA (SIEN), se determinaron las medias del índice $Z$ talla/edad) niños a los 6 y 12 meses de los distritos de Huánuco con puntajes similares en vulnerabilidad a la desnutrición crónica donde se evidencio una reducción de 0,29 (de $-0,91 \pm 1,18$ a $-1,20 \pm 1,13$ ) del puntaje $Z$ de talla/edad e incluso al incluir a todos los niños del departamento de Huánuco se encontró que la caída del puntaje $Z$ talla/edad fue de 0,22 (de $-0,64 \pm 1,21$ a $-0,86 \pm 1,16)$ en el mismo período del estudio. Desde esa perspectiva el consumo de LNS podría tener un efecto protector al atenuar en casi $50 \%$ la tendencia a la caída del indicador talla/edad, en niños de seis a once meses de edad en ámbitos similares al del estudio.

En el Perú se ha evaluado el efecto de otros suplementos nutricionales como los MNP que proveen hierro y cinco micronutrientes. El estudio de Huamán-Espino et al. realizado en niños de 6 a 35 meses no encontró efectividad del MNP sobre la reducción de la prevalencia de anemia o mejora en los niveles de hemoglobina en el departamento de Apurímac ${ }^{(10)}$. Otro estudio del impacto de MNP sobre anemia en niños de seis a once meses de edad mostró una reducción de 33 puntos porcentuales en anemia al final del seguimiento ${ }^{(26)}$. Sin embargo, este estudio solo se analizó al $57 \%$ de la muestra original por lo que los resultados podrían tener algún sesgo de atrición, dada la pérdida del $42 \%$ de los niños en estudio ${ }^{(27)}$.

Otro resultado importante que resaltar es la alta adherencia, aceptabilidad y pocas molestias reportadas que generó el consumo del LNS, estos resultados también han sido observados en otro estudio en Guatemala ${ }^{(14)}$ a diferencia de otros suplementos como sulfato ferroso o MNP, el LNS es un alimento listo para consumir, no requiere agua y aporta un $20 \%$ de la energía requerida por el niño, así como 19 nutrientes.

Una limitación del estudio es que no se estableció un grupo control para comparar los resultados, dado que se trató de un estudio preexperimental; este diseño potencialmente puede tener sesgos que atenten contra la validez interna: historia, maduración biológica, realización de pruebas, instrumentación y efecto de regresión ${ }^{(28)}$. Al respecto, se debe mencionar que la primera fuente de invalidación es la historia, es decir, la ocurrencia de otros eventos simultáneos que puedan modificar los resultados, se reduce cuando las intervenciones son de corto plazo y cuando la cadena causal entre la intervención y el resultado esperado es directa ${ }^{(16)}$; en este estudio la intervención fue por 6 meses y existe plausibilidad biológica y antecedentes del efecto del LNS sobre la anemia y crecimiento lineal. En cuanto a maduración biológica, se debe considerar que los niños participantes fueron de seis a once meses de edad, período en el que se mantienen uniformes los requerimientos de hierro en los niños ${ }^{(29)}$ por lo que el sesgo de maduración biológica sería mínimo. Por otro lado, respecto a realización de pruebas e instrumentación, conviene recordar que se producen sobre todo en pruebas subjetivas como test de conocimientos, psicológicos (16), en este estudio se realizaron mediciones objetivas de las variables principales como determinación de hemoglobina y medición de peso y talla. Sin embargo, existen otros sesgos inherentes al diseño usado que no se pudieron controlar en la estimación final como la selección, regresión a la media y otros de validez externa.

Otro aspecto limitante que mencionar es el tamaño de muestra que resultó insuficiente para verificar las hipótesis de mejora en desnutrición aguda y global a pesar de que se observa que hubo mejoras del $50 \%$. Por otra parte, dentro del estudio no se exploraron variables relacionadas a alimentación infantil de los niños suplementados como duración de lactancia materna exclusiva, consumo de otros alimentos fuentes de hierro; al respecto, cabe precisar que el desarrollo de la anemia está determinado por el aumento de los requerimientos nutricionales de hierro en esta edad $y$, si bien los alimentos (incluida la leche materna), pueden aportar hierro, el porcentaje de absorción y la cantidad de hierro en la dieta tienen una pequeña influencia para evitar la anemia ${ }^{(30)}$.

En conclusión, los resultados del estudio muestran el efecto de la suplementación con LNS para mejorar los niveles de hemoglobina y reducir la prevalencia de anemia en niños menores de doce meses, por lo que podría constituir una alternativa efectiva para prevenir y controlar la anemia infantil. Se requiere realizar ensayos controlados y aleatorizados para evaluar apropiadamente la efectividad del LNS sobre la anemia y crecimiento lineal.

Agradecimientos: a los directivos y personal de salud de la Dirección Regional de Salud Huánuco, Red de Salud Huánuco y las Microrredes de Salud Huacar y San Rafael por su importante participación en la implementación de este estudio.

Conflictos de interés: los autores declaran no tener conflictos de interés.

Fuentes de financiamiento: el estudio se realizó con fondos de UBS Optimus Foundation y de la Fundación Acción Contra el Hambre; los suplementos de LNS-Nutributter $₫$ fueron donados por la Empresa NUTRISET. 


\section{REFERENCIAS BIBLIOGRÁFICAS}

1. Black RE, Victora CG, Walker SP, Bhutta ZA, Christian P, de Onis M, et al. Maternal and child undernutrition and overweight in low-income and middle-income countries. Lancet. 2013 Aug 3;382(9890):427-51. doi: 10.1016/S0140-6736(13)60937-X.

2. Victora CG, Adair L, Fall C, Hallal PC, Martorell R, Richter L, et al. Maternal and child undernutrition: consequences for adult health and human capital. Lancet. 2008 Jan 26;371(9609):340-57. doi: 10.1016/ S0140-6736(07)61692-4.

3. Black RE, Allen LH, Bhutta ZA, Caulfield LE, de Onis M, Ezzati M, et al. Maternal and child undernutrition: global and regional exposures and health consequences. Lancet. 2008 Jan 19;371(9608):243-60. doi: 10.1016/S0140-6736(07)61690-0.

4. Paraje G. Desnutrición crónica infantil y desigualdad socioeconómica en América Latina y el Caribe. Rev CEPAL. 2009;99:43-69.

5. Instituto Nacional de Estadística e Informática. Encuesta Demográfica y de Salud Familiar 2007. Lima: INEI; 2008

6. Instituto Nacional de Estadística e Informática. Encuesta Demográfica y de Salud Familiar 2014. Reporte del primer semestre. Lima: INEI; 2014.

7. Sobrino M, Gutiérrez C, Cunha AJ, Dávila M, Alarcón J. Desnutrición infantil en menores de cinco años en Perú: tendencias y factores determinantes. Rev Panam Salud Publica. 2014;35(2):104-12.

8. Aparco JP, Huamán-Espino L, Pillaca J. Cambios en magnitud y tendencias de la desnutrición crónica en el Perú, análisis del periodo 1996 a 2011 . Rev Peru Epidemiol. 2012;16(3):1-6.

9. Instituto Nacional de Estadística e Informática. Encuesta Demográfica y de Salud Familiar 2011. Lima: INEI; 2012

10. Huamán-Espino L, Aparco JP, NuñezRobles E, Gonzáles E, Pillaca J, MaytaTristán P. Consumo de suplementos con multimicronutrientes Chispitas y anemia en niños de 6 a 35 meses: estudio transversal en el contexto de una intervención poblacional en Apurímac, Perú. Rev Peru Med Exp Salud Publica. 2012;29(3):314-23.
11. Adu-Afarwuah S, Lartey A, Brown KH, Zlotkin S, Briend A, Dewey KG. Randomized comparison of 3 types of micronutrient supplements for home fortification of complementary foods in Ghana: effects on growth and motor development. Am J Clin Nutr. 2007. Aug;86(2):412-20.

12. Huybregts L, Houngbé F, Salpéteur C, Brown R, Roberfroid D, Ait-Aissa M, et al. (2012) The effect of adding ready-to-use supplementary food to a general food distribution on child nutritional status and morbidity: a cluster-randomized controlled trial. PLoS Med 2012;9(9):e1001313. doi: 10.1371/journal.pmed.1001313.

13. Dewey KG, Arimond M. Lipid-based nutrient supplements: how can they combat child malnutrition?. PLoS Med. 2012;9(9):e1001314. doi: 10.1371/journal.pmed.1001314.

14. Matías S, Chaparro C, Perez A, Peerson J, Dewey K. Acceptability of a Lipid-Based Nutrient Supplement among Guatemalan Infants and Young Children. Washington, DC: FANTA; 2011.

15. Arteaga R; Vilcarromero M. Estudio de Aceptabilidad de los productos Nutributter y QBmix de NUTRISET con niños y niñas menores de 5 años, madres gestantes y lactantes, y familias en situación de pobreza. ACH; 2010.

16. Shadish WR, Cook TD, Campbell DT. Experimental and quasiexperimental designs for generalized causal inference. Boston, MA: Houghton Mifflin Company; 2002.

17. Programa Mundial de Alimentos. Mapa de vulnerabilidad a la desnutrición crónica desde la perspectiva de la pobreza. Lima: PMA; 2007.

18. Jordán T. Guía Técnica: procedimiento para la determinación de hemoglobina mediante hemoglobinómetro portátil. Lima: MINSA-INS; 2013.

19. Contreras M, Valenzuela R. La medición de la talla y el peso; guía para el personal de la salud del primer nivel de atención. Lima: MINSAINS; 2004.

20. De Onis M, Garza C, Onyango AW, Martorell R (ed). Relationship between physical growth and motor development in the WHO Child Growth Standards. Acta Paediatr.2006;95 supl 450:96-101.

21. Organización Mundial de la Salud: el uso clínico de la sangre en medicina general, Obstetricia, Pediatría y Neonatología, cirugía y anestesia, trauma y quemaduras. Ginebra: OMS; 2001.

22. Iannotti LL, Dulience SJ, Green J, Joseph S, François J, Anténor ML, et al. Linear growth increased in young children in an urban slum of Haiti: a randomized controlled trial of a lipidbased nutrient supplement. Am J Clin Nutr. 2014 Jan;99(1):198-208. doi: 10.3945/ajen.113.063883.

23. Phuka JC, Maleta K, Thakwalakwa $\mathrm{C}$, Cheung $\mathrm{YB}$, Briend A, Manary MJ, et al. Complementary feeding with fortified spread and incidence of severe stunting in 6- to 18-monthold rural Malawians. Arch Pediatr Adolesc Med. 2008 Jul;162(7):61926. doi: 10.1001/archpedi.162.7.619.

24. Thakwalakwa C, Ashorn P, Phuka J, Cheung YB, Briend A, Puumalainen $\mathrm{T}$, et al. A lipid-based nutrient supplement but not corn-soy blend modestly increases weight gain among 6 - to 18-month-old moderately underweight children in rural Malawi. J. Nutr. 2010 Nov;140(11):2008-13. doi: $10.3945 /$ jn.110.122499.

25. Adu-Afarwuah S, Lartey A, Brown $\mathrm{KH}$, Zlotkin S, Briend A, Dewey KG. Home fortification of complementary foods with micronutrient supplements is well accepted and has positive effects on infant iron status in Ghana. Am J Clin Nutr. 2008 Apr;87(4):929-38.

26. Munayco CV, Ulloa-Rea ME, MedinaOsis J, Lozano-Revollar CR, Tejada V, Castro-Salazar C, et al. Evaluación del impacto de los multimicronutrientes en polvo sobre la anemia infantil en tres regiones andinas del Perú. Rev Peru Med Exp Salud Publica. 2013;30(2):229-34.

27. Aparco JP, Huamán-Espino L. Comentarios al artículo: Evaluación del impacto de los multimicronutrientes en polvo sobre la anemia infantil en tres regiones andinas del Perú. Rev Peru Med Exp Salud Publica. 2013;30(4):725-6. 
28. Campbell DT, Stanley JC. Experimental and Quasi-experimental Designs for Research. Chicago: Rand McNally; 1966.

29. Yip R, Dallman P. Hierro. En: Bowman B, Rusell R. Conocimientos Actuales en Nutrición. 8va ed.
Washington, DC: OPS/ILSI; 2001. p. 340-59.

30. Allen L, Casterline-Sabel J. Prevalence and causes of nutritional anemias. En: Ramakrishnan U. Nutritional Anemias. Florida: CRC Press LLC; 2001. p. 7-22.
Correspondencia: Alejandro Vargas Vásquez Dirección: Calle Santa Isabel 263, 2. ${ }^{d o}$ piso Miraflores

Teléfono: +5116282835

Correo electrónico:avargas@pe.acfspain.org

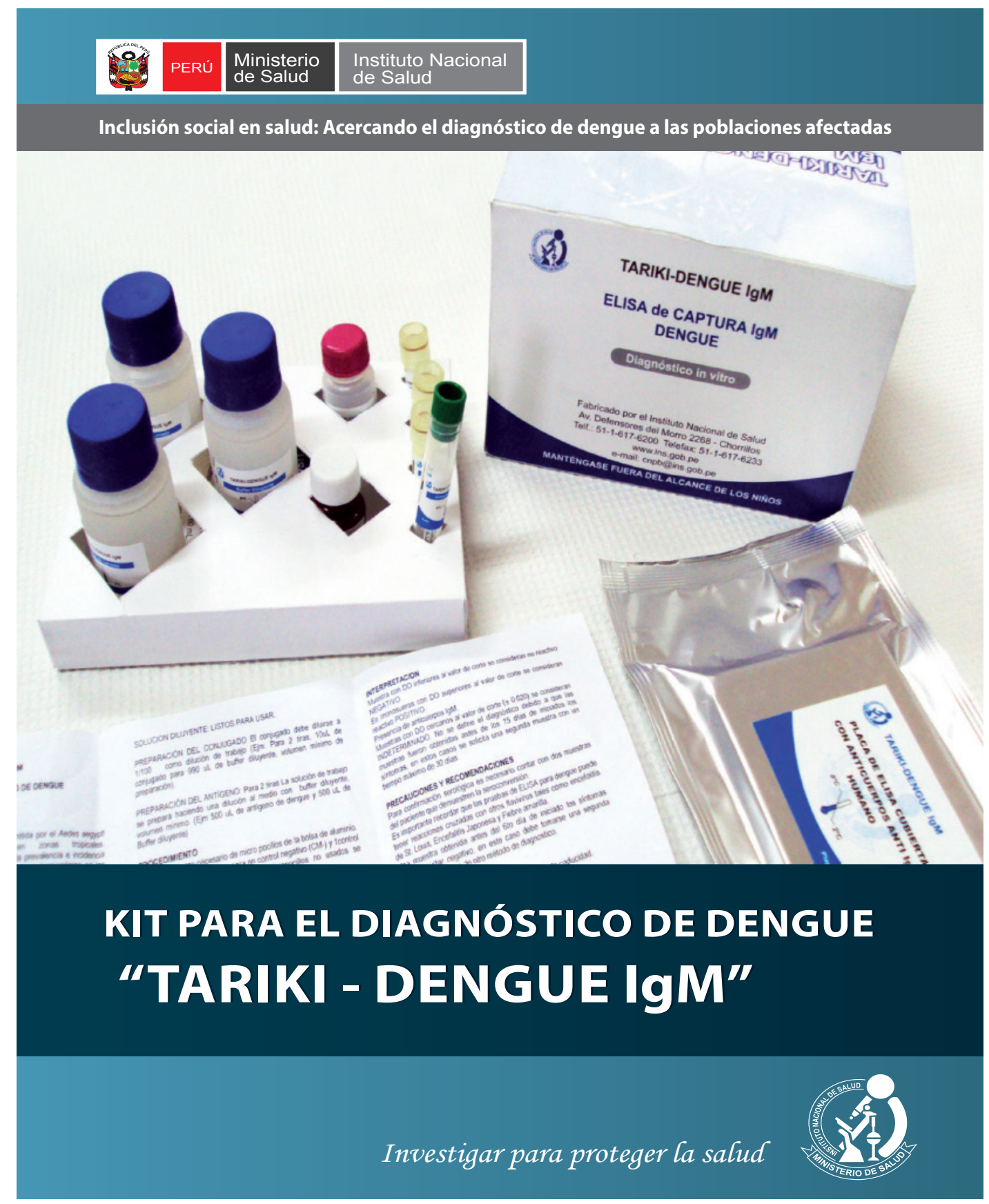

